\title{
The Evaluation of Degeneration of Posterior Cruciate Ligament Using CT Hounsfield Unit in Knee Osteoarthritis
}

\section{Yoshikazu Sumida ( $\sim$ mpbpf007@yahoo.co.jp )}

Hiroshima University

\section{Tomoyuki Nakasa}

Hiroshima University

\section{Masakazu Ishikawa}

Hiroshima University

\section{Atsuo Nakamae}

Hiroshima University

Nobuo Adachi

Hiroshima University

\section{Research article}

Keywords: Degeneration, PCL, Osteoarthritis, CT scan, Hounsfield unit

Posted Date: September 10th, 2020

DOI: https://doi.org/10.21203/rs.3.rs-44938/v2

License: (c) (1) This work is licensed under a Creative Commons Attribution 4.0 International License. Read Full License

Version of Record: A version of this preprint was published on March 26th, 2021. See the published version at https://doi.org/10.1186/s12891-021-04177-w. 


\section{Abstract}

Background: The degeneration of posterior cruciate ligament (PCL) is often seen in knee osteoarthritis $(\mathrm{OA})$, however there is no established method for its evaluation. The purpose of this study is to investigate whether Hounsfield unit (HU) using CT (computed tomography) could be a useful scale to evaluate the degeneration of PCL in knee OA.

Methods: Knee OA treated with total knee arthroplasty (21 patients, 21 knees) and non-osteoarthritic knees (21 patients, 21 knees) were prospectively studied. All PCLs in the knees were analyzed by CT scan. PCL in sagittal section was divided into three regions: proximal, middle and distal portions. The HU value of PCL at each area was measured. In osteoarthritic knees, tissues from PCL were collected and histologically graded.

Results: The HU value in $\mathrm{OA}$ and non-OA was 70.7 and $88.4 \mathrm{HU}(\mathrm{p}<0.05)$ at proximal region, $75.7 \mathrm{HU}$ and $85.3(p<0.05)$ at central region and 82.3 and $86.5 \mathrm{HU}(p>0.05)$ at distal region, respectively. The degeneration of PCL was graded as follows; mild: 1 case, moderate: 3 cases and severe: 17 cases at proximal portion and mild: 16 cases, moderate: 4 cases and severe: 1 case at distal portion. The HU value was $84.5 \mathrm{HU}$ for mild, $72.1 \mathrm{HU}$ for moderate and $70.6 \mathrm{HU}$ for severe (mild versus moderate: $p<0.05$, mild versus severe: $p<0.05$, moderate versus severe: $p>0.05$ ).

Conclusions: In knee OA, lower HU value in PCL indicates progression of degeneration. The CT HU value could be a useful measurement to predict the grade of degeneration of PCL.

\section{Background}

Knee osteoarthritis $(\mathrm{OA})$ is one of the most common diseases we encounter in daily practice. Although the pathogenesis of knee osteoarthritis is not still elucidated, it is known that the degeneration of PCL (posterior cruciate ligament) is one of the features seen in the process of knee OA [1]. Magnetic resonance imaging (MRI) has been commonly used in order to evaluate the degeneration of PCL. However, MRI is only useful to detect rupture, mucoid degeneration and ganglion in PCLs and it is not enough to analyze the quality within the ligaments which have a short echo time [2]. On the other hand, computed tomography (CT) scan is also one of the conventional modalities to assess knee OA. CT is excellent not only for depicting cortical bone and soft tissue calcifications [3], but also for the assessment of soft tissues [4]. It offers better visualization of subchondral bone cysts and osteophytes compared with MRI and radiography, and also helps surgical planning preoperatively [5]. There is no previous literature mentioned about the evaluation of PCL by using CT, however it is reported that CT scan makes it possible to assess soft tissues and materials quantitatively by using Hounsfield unit (HU) [4]. $\mathrm{HU}$ is also known as CT numbers which corresponds with average amount of radiation absorbed by the tissue [6]. Therefore, we recognized HU value could have potential to analyze the quality of PCL. We hypothesized that the PCL degeneration is relevant to $\mathrm{CT}$ HU value, which provide information about its property. The 
purpose of this study is to investigate whether CT HU value could be a useful scale to evaluate the degeneration of PCL in knee osteoarthritis.

\section{Methods}

\section{Subjects}

Twenty-one osteoarthritic (OA) knees (21 patients) treated with total knee arthroplasty (TKA) in our hospital from 2016 to 2018 were prospectively evaluated. All of the OA knees met American College of Rheumatology (ACR) Clinical Classification Criteria for Osteoarthritis of the knee [7]. As control group, 21 non-osteoarthritic knees (21 patients) with soft tissue tumor (nine knees), bone tumor (nine knees) and osteoarthritic ankle (three knees) were compared. The gender distribution was 1 male and 20 females in OA group and 10 males and 11 females in control group. The average age was 73.5 years with a range of 66 - 81 years in OA group and 73.5 years with a range of $58-90$ years in control group. Inclusion criteria for knee OA was primary medial knee OA without any previous history of knee injury before surgery. Rheumatoid arthritis and other inflammatory arthritis were excluded. The average body mass index was $25.8 \pm 3.7 \mathrm{~kg} / \mathrm{m}^{2}$ in $\mathrm{OA}$ group and $22.5 \pm 3.4 \mathrm{~kg} / \mathrm{m}^{2}$ in control group respectively ( $\left.\mathrm{P} \otimes 0.05\right)$. According to the radiological classification by Kellgren and Lawrence [8], nine knees were classified as grade 3 and 12 knees as grade 4 in OA group, respectively, and all 21 knees were classified as grade 0 in control group. The average femorotibial angle was $183.2^{\circ} \pm 8.4^{\circ}$ in $\mathrm{OA}$ group and $175.6^{\circ} \pm 1.3^{\circ}$ in control group, respectively $(\mathrm{P} \otimes 0.05)$. All patients underwent $\mathrm{CT}$ scans preoperatively and PCLs were evaluated quantitatively using HUs. At the time of TKA, $3 \mathrm{~mm} \times 3 \mathrm{~mm} \times 3 \mathrm{~mm}$ large biopsy from proximal and distal section of PCL was performed respectively. Collected tissues were microscopically analyzed and the results were compared with those obtained from CT scans. Institutional Review Board (IRB) of Hiroshima University hospital approved this study and all procedures were performed under the informed consent from all the patients.

\section{Evaluation in CT scans}

All patients were scanned with Aquilion One (CANON MEDICAL SYSTEMS CORPORATION, JAPAN). The scanning parameters were as follows: $120 \mathrm{kV}, 150 \mathrm{mAs}$ and $1.5 \mathrm{~mm}$ slice thickness. Images were analyzed in a workstation, AZE VirtualPlace Ver. 4.7 (AZE, Japan). Patients were asked to fix their knees in extension. All CT scans were taken with sagittal plane oriented almost parallel to the longitudinal axis of PCL. An image demonstrating the center of PCL was picked up, and the PCL was divided into three parts: proximal, middle and distal sections (Fig. 1). We measured the $\mathrm{HU}$ value in a region of interest (ROI), a $3.64 \mathrm{~mm}^{2}$ circle, in PCLs. The measurement was performed in three random plots in each section and the average $\mathrm{HU}$ value from these three plots was calculated.

\section{Microscopic analysis of PCLs}

At the time of TKA, samples were carefully resected from proximal and distal portions of PCLs, respectively. The samples were fixed in $4 \%$ paraformaldehyde phosphate-buffered saline (Wako Pure 
Chemical Industries Ltd., Osaka, Japan) immediately after collection. 24 hours later, they were embedded in paraffin and cut into $5 \mu \mathrm{m}$-thick sections. The sections were stained with Safranin-0 fast-green and evaluated microscopically. We evaluated the specimens at 100x in the examination of ten fields according to the method described in previous literatures [9].The grade of collagen fiber impairment was classified as follows: mild, impairment of less than $20 \%$ of the collagen fibers; moderate, impairment of at least $20 \%$ and less than $50 \%$ of the collagen fibers; severe, impairment of more than $50 \%$ of the collagen fiber (Fig. 2).

\section{Statistical Analysis}

Data were analyzed using the Statistical Package for Statcel 4 (OMS, Saitama, Japan). All measured values were expressed as means \pm standard deviation (SD). Mann-Whitney test and KruskalWallis test were used for comparisons between 2 groups and 3 groups, respectively. Pख0.05 was considered for statistical significance.

\section{Results}

Quantitative comparison of CT HU value maps demonstrated that OA group had lower HU value (Fig. 3). At the proximal portion, the CT HU value was $70.7 \pm 9.8 \mathrm{HU}$ in $\mathrm{OA}$ group and $88.4 \pm 4.7 \mathrm{HU}$ in control group, respectively, and there was a significant difference between two groups (Pख0.05). At the middle portion, it was $75.7 \pm 11.7 \mathrm{HU}$ in $\mathrm{OA}$ group and $85.3 \pm 4.5 \mathrm{HU}$ in control group, and there was a significant difference between two groups (Pख0.05). At the distal portion, it was $82.3 \pm 14.1 \mathrm{HU}$ in OA group and 86.5 $\pm 5.1 \mathrm{HU}$ in control group, and there was no significant difference between 2 groups $(P>0.05)($ Table. 1$)$.

In OA group, microscopic analysis of the PCL in OA knee revealed that severe degeneration in 17 cases $(81.0 \%)$, moderate degeneration in three cases $(14.2 \%)$ and mild degeneration in one case $(4.76 \%)$ in the proximal portion and severe degeneration in one case $(4.76 \%)$, moderate degeneration in four cases (19.0\%) and mild degeneration in 16 cases (76.2\%) in the distal portion (Fig. 4).

The CT HU value of PCL with severe degeneration was $70.6 \pm 10.7 \mathrm{HU}$, that with moderate degeneration was $72.1 \pm 9.50 \mathrm{HU}$ and that with mild degeneration was $84.5 \pm 13.7 \mathrm{HU}$. The CT HU value of mild degeneration was significantly smaller than that of severe and moderate degeneration (Pख0.05) (Fig. 5).

\section{Discussion}

The most important finding in this study is that we demonstrated that the HU value of PCL could reflect the grade of degeneration. Also, we revealed that the PCL degeneration in knee OA was severer at proximal portion.

In our study, the $\mathrm{HU}$ value in the PCL with mild degeneration was $84.5 \mathrm{HU}$ which is significantly more than $70.6 \mathrm{HU}$ and $72.1 \mathrm{HU}$ in severe and moderate degeneration, respectively. As long as we know, this is the first literature demonstrated the HU value of PCL in knee OA. Woodard et al. and Willner et al. revealed the 
typical HU values for various tissues in the literatures: tendon; $101.6 \mathrm{HU}$, muscle; $39.4 / 40.4 \mathrm{HU}$, adipose; -30.6/-66.6 HU, for instance [10]. However, they did not mention about the HU of ligamentous tissue. There are some reports described the utility of CT HU for the evaluation of soft tissues. Byun et al. found a direct correlation between the inflammatory activity and the density of extraocular fat and lacrimal gland by using HUs in patients with thyroid-associated orbitopathy, and they concluded that the density could predict active inflammation [3]. Another report by Chikui et al. revealed Lipoma had a specific range of CT HU values and displaced the surrounding soft tissue [11]. They also suggested that it is expected that myxoid and pleomorphic sarcomas could easily be differentiated from lipomas because the CT values would be higher than those of adipose tissue. In another literature, Spruit et al. showed HU value of bone graft in intervertebral metal-cage contents increased in the postoperative period, which demonstrated biological activity and change of bone mineral content of the tissue [12]. Barber et al. evaluated the long-term in vivo degradation of biocomposite interference screw with $\mathrm{HU}$ value and confirmed its osteoconductivity [13]. He also analyzed the density of the synthetic multiphase implant for the donor site autologous osteochondral transplantation in another literature. The density declined over time to that of fibrous scar, which revealed no evidence of bone ingrowth, osteoconductivity and ossification of the implant [12].According to these previous reports, the HU value using CT scans could provide us objective and quantitative evaluation for soft tissues and help us predict properties of those tissues.

Another finding in this study was that PCL in knee OA showed severer degenerative changes in proximal portion with lower $\mathrm{HU}$ value compared with that in distal portion. Levy et al. demonstrated that the first changes observed in the PCL were fiber disorganization, mucoid and chondroid metaplasia [14]. The study by Kumagai et al. indicated that chondroid metaplasia is associated with the progression of degeneration in human ligaments [15]. The lower HU value in proximal portion of the PCL could reflect those histological changes. They evaluated PCL by scoring inflammation, mucoid degeneration, chondroid metaplasia, cystic changes other than orientation of collagen fiber. On the other hand, we evaluated the degeneration only in terms of collagen fiber impairment. Viidik demonstrated that changes in collagen fibrils affect the biomechanical properties of the ligament [16]. Therefore, we believe that the impairment of collagen fibers is the most affective factor from the aspect of ligamentous function and evaluated it microscopically.

Severer degenerative change was more likely to be seen in proximal portion of PCL in our study. Although Levy et al. and Kumagai et al. described PCL degeneration with histological evaluation, they analyzed the tissue resected from middle portion and proximal one third of the PCL, respectively [14]. There is no study demonstrated the detailed emergence of degeneration in PCL. It is reported that the degeneration in ACL occurs from proximal portion [17], however the mechanism and pathogenesis of PCL degeneration is still unknown and should be elucidated in the future.

Aggarwal et al. showed the Knee Society Score, anteroposterior instability, ACL appearance and erosion in the lateral tibiofemoral compartment are predictors of PCL degeneration, however they are not enough information to assess the property of PCL quantitatively [1]. On the other hand, the HU value using CT 
scans is shown as a number objectively which reflects its property, therefore it could be a new scale to predict the degeneration of PCL.

The most important finding in this study is that we revealed the potential to predict the property of PCL using CT HU value. Even if macroscopic appearance of PCL is normal, degenerative change is observed microscopically [18]. Therefore, it is profitable to know the grade of degeneration in PCL with the CT scan. Considering the fact that MRI is not useful enough to analyze the quality within the ligaments which have a short echo time [2], the measurement of CT HU value could be a valid method to provide us more details about the ligament property. Although further studies are required, the evaluation using the CT HU value could be applied not only for PCL but also for other soft tissues.

There are some limitations in this study. Firstly, the sample size was small and we need large population for both groups. Secondly, the gap of the number of patients with different gender in both OA and control groups. More women were included in OA group and more men in control group. The influence of gender was not considered in this study. Thirdly, one sagittal image from CT scans might not be enough to correspond to the histological result of the obtained sample. Lastly, bone or soft tissue tumor might have affected the property of PCL. However, they were the best control group that we could obtain, because it is ethically difficult to have healthy people undergo CT scans considering the exposure to radiation.

\section{Conclusions}

In this study, we revealed the utility of the $\mathrm{HU}$ value using CT scans for the evaluation of degenerated PCL in knee OA. The value decreased as the degenerative changes proceeded. The CT HU value could be a useful measurement to predict the grade of degeneration of PCL.

\section{Declarations}

\section{Ethics approval and consent to participate}

The study was approved by the Ethics Committee of Hiroshima university hospital and written consent was obtained from all patients.

\section{Consent for publication}

Not applicable

\section{Availability of data and materials}

The datasets used and analysed during the current study are available from the corresponding author on reasonable request.

\section{Competing interests}


The authors declare that they have no competing interests.

\section{Funding}

This research did not receive any specific grant from funding agencies int the public, commercial, or notfor-profit sectors.

\section{Author's contributions}

Dr.YS and TN take responsibility for the design of this study. YS and TN analysed and interpreted the patient data. $\mathrm{MI}$ and $\mathrm{AN}$ reviewed and edited the manuscript. TN and NA contributed to the critical revision of the article. All authors read and approved the final manuscript.

\section{Acknowledgements}

We appreciate Minoru Ishifuro for the technical support.

\section{Authors' information}

${ }^{1}$ Department of Orthopaedic Surgery, Graduate School of Biomedical and Health Sciences, Hiroshima University, Japan

\section{Conflict of interest}

The authors declare that they have no conflict of interest.

\section{Ethical approval}

This study was conducted in compliance with the Declaration of Helsinki and was approved by our institutional review board at our hospital.

\section{Abbreviations}

Osteoarthlitis (OA), Posterior cruciate ligament $(\mathrm{PCL})$, Magnetic resonance imaging (MRI), CT (computed tomography), Hounsfield unit (HU)

\section{References}

1. Aggarwal AK, Goel A, Radotra BD. Predictors of Posterior Cruciate Ligament Degeneration in Osteoarthritic Knees. Journal of Orthopaedic Surgery. 2013;21:15-8.

2. Draghi F, Bortolotto C, Coscia DR, Canepari M, Gitto S. Magnetic resonance imaging of degenerative changes of the posterior cruciate ligament. Acta Radiol. 2017;58:338-43.

3. Byun JS, Moon NJ, Lee JK. Quantitative analysis of orbital soft tissues on computed tomography to assess the activity of thyroid-associated orbitopathy. Graefes Arch Clin Exp Ophthalmol. 
2017;255:413-20.

4. Barber FA, Dockery WD. A computed tomography scan assessment of synthetic multiphase polymer scaffolds used for osteochondral defect repair. Arthroscopy. 2011;27:60-4.

5. Wenham CYJ, Grainger AJ, Conaghan PG. The role of imaging modalities in the diagnosis, differential diagnosis and clinical assessment of peripheral joint osteoarthritis. Osteoarthr Cartil. 2014;22:1692-702.

6. Ai HA, Meier JG, Wendt RE. HU deviation in lung and bone tissues: Characterization and a corrective strategy. Med Phys. 2018;45:2108-18.

7. Altman R, Asch E, Bloch D, Bole G, Borenstein D, Brandt K, et al. Development of criteria for the classification and reporting of osteoarthritis. Classification of osteoarthritis of the knee. Diagnostic and Therapeutic Criteria Committee of the American Rheumatism Association. Arthritis Rheum. 1986;29:1039-49.

8. Kellgren JH, Lawrence JS. Radiological assessment of osteo-arthrosis. Ann Rheum Dis. 1957;16:494-502.

9. Martins GC, Camanho G, Rodrigues MI, Filho LFM, Demange MK. Histopathological analysis of the posterior cruciate ligament in primary osteoarthritis. Eur J Orthop Surg Traumatol. 2018;28:691-9.

10. Willner M, Fior G, Marschner M, Birnbacher L, Schock J, Braun C, et al. Phase-Contrast Hounsfield Units of Fixated and Non-Fixated Soft-Tissue Samples. PLoS ONE. 2015;10:e0137016.

11. Chikui T, Yonetsu K, Yoshiura K, Miwa K, Kanda S, Ozeki S, et al. Imaging findings of lipomas in the orofacial region with CT, US, and MRI. Oral Surg Oral Med Oral Pathol Oral Radiol Endod. 1997;84:88-95.

12. Spruit M, Meijers H, Obradov M, Anderson PG. CT density measurement of bone graft within an intervertebral lumbar cage: increase of hounsfield units as an indicator for increasing bone mineral content. J Spinal Disord Tech. 2004;17:232-5.

13. Barber FA, Dockery WD. Long-Term Degradation of Self-Reinforced Poly-Levo (96\%)/Dextro (4\%)Lactide/ $\beta$-Tricalcium Phosphate Biocomposite Interference Screws. Arthroscopy. 2016;32:608-14.

14. Levy YD, Hasegawa A, Patil S, Koziol JA, Lotz MK, D'Lima DD. Histopathological changes in the human posterior cruciate ligament during aging and osteoarthritis: correlations with anterior cruciate ligament and cartilage changes. Ann Rheum Dis. 2013;72:271-7.

15. Kumagai K, Sakai K, Kusayama Y, Akamatsu Y, Sakamaki K, Morita S, et al. The extent of degeneration of cruciate ligament is associated with chondrogenic differentiation in patients with osteoarthritis of the knee. Osteoarthr Cartil. 2012;20:1258-67.

16. Viidik A, Danielson CC, Oxlund H. On fundamental and phenomenological models, structure and mechanical properties of collagen, elastin and glycosaminoglycan complexes. Biorheology. 1982;19:437-51.

17. Hasegawa A, Otsuki S, Pauli C, Miyaki S, Patil S, Steklov N, et al. Anterior cruciate ligament changes in the human knee joint in aging and osteoarthritis. Arthritis Rheum. 2012;64:696-704. 
18. Allain J, Goutallier D, Voisin MC. Macroscopic and histological assessments of the cruciate ligaments in arthrosis of the knee. Acta Orthop Scand. 2001;72:266-9.

\section{Table}

Table.1 The average CT HU value \pm standard deviation of PCL from each region.

The value was significantly lower at proximal and middle portion of PCL. (**p $<0.01$, Mann-Whitney test)

\begin{tabular}{lll}
\hline & Control & OA \\
\hline proximal & $88.4 \pm 4.7$ & $70.7 \pm 9.8^{* *}$ \\
middle & $85.3 \pm 4.5$ & $75.7 \pm 11.7^{* *}$ \\
distal & $86.5 \pm 5.1$ & $82.3 \pm 14.1$ \\
\hline
\end{tabular}

\section{Figures}




\section{proximal}

\section{middle}

\section{distal}

\section{Figure 1}

Sagittal image of PCL. PCL is divided into three regions, distal, middle, proximal portions. HU in a region of interest (ROI), a $3.64 \mathrm{~mm} 2$ circle was measured. The average $\mathrm{HU}$ from three randomly chosen ROI was calculated. 

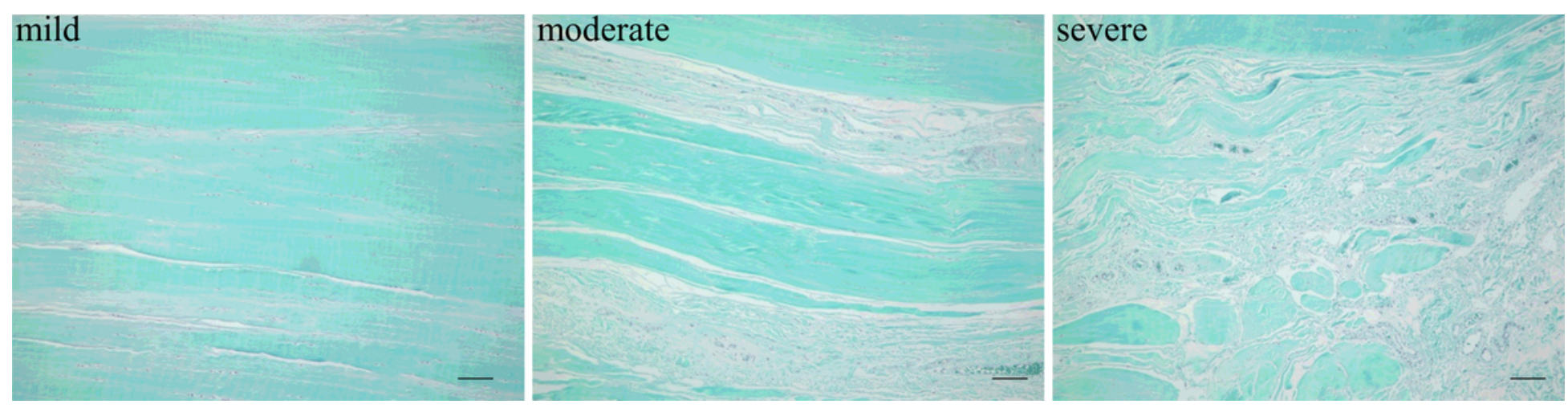

Figure 2

Collagen fibers in PCL stained with Safranin-O fast-green. The grade of collagen fiber impairment was classified as follows: mild, impairment of less than $20 \%$ of the collagen fibers; moderate, impairment of at least $20 \%$ and less than $50 \%$ of the collagen fibers; severe, impairment of more than $50 \%$ of the collagen fiber. The scale bar indicates $100 \mu \mathrm{m}$.
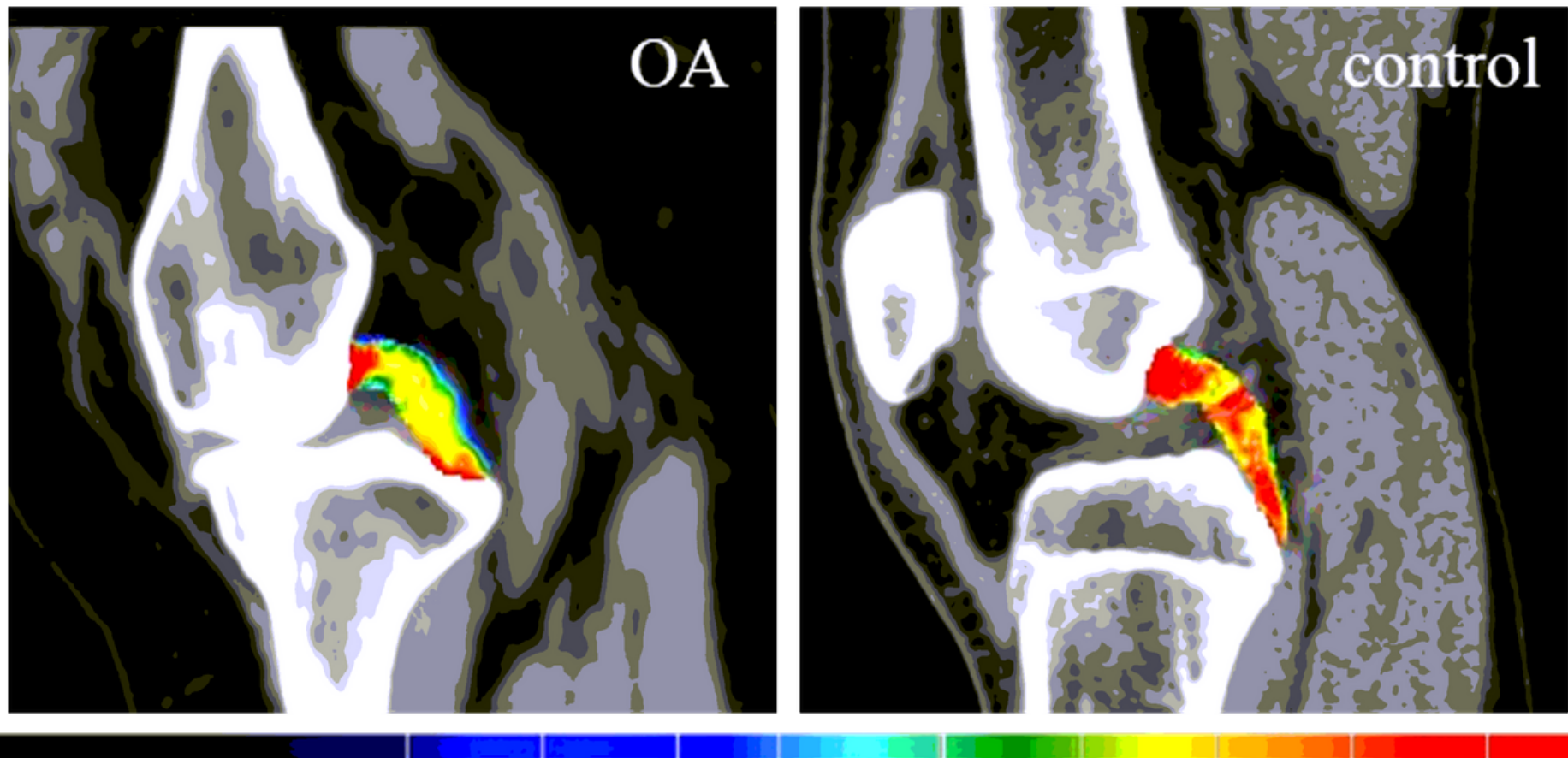

\section{Figure 3}

Quantitative comparison of CT HU value maps between OA and control group. The image of OA shows lower CT HU value in the PCL. 
Degeneration at proximal portion

$$
\text { mild }
$$

1 case

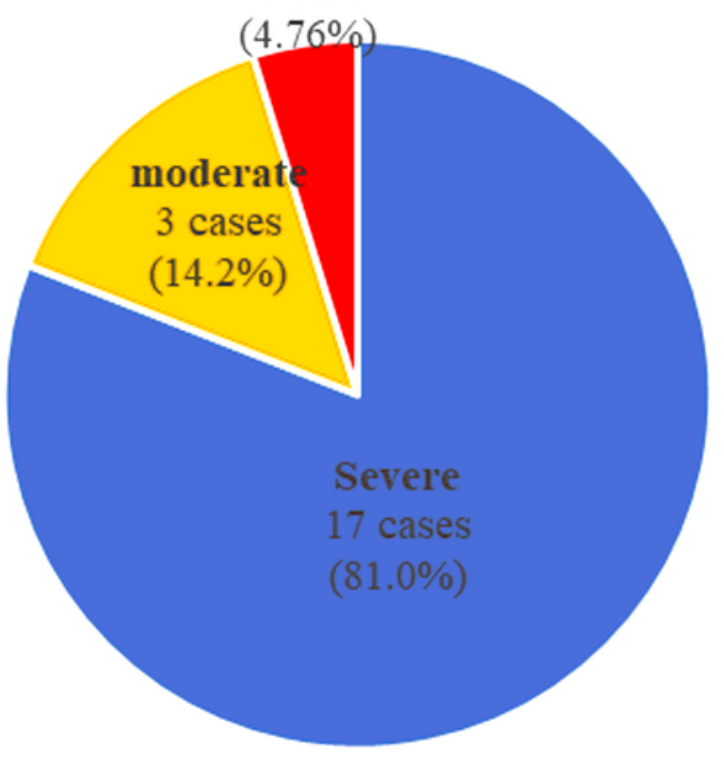

Degeneration at distal portion severe

1 case

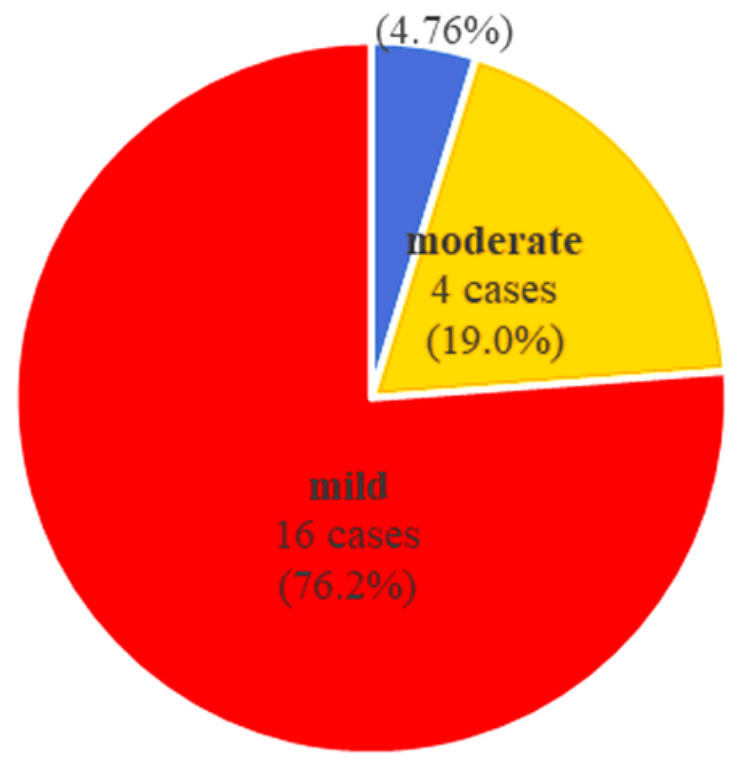

Figure 4

The number and ratio with different grade of degeneration at proximal and distal portions. More severe cases were seen at proximal portion compared with distal portion.

$(\mathrm{HU})$
110
100
90
80
70
60
50
40
30
20
10
0

\section{CT HU value of degenerated PCL}

*

* 


\section{Figure 5}

The CT HU values for PCL with different degenerative grade are shown. The CT HU value of mild degeneration was significantly higher than the others (mild versus moderate: ${ }^{*} p<0.05$, mild versus severe: ${ }^{*} p<0.05$, moderate versus severe: $p>0.05$, Kruskal-Wallis test). The error bar shows standard deviation. 